\title{
Qualidade das águas do Rio Jaguari e relações entre parâmetros: um estudo de Redes Complexas
}

\author{
Fernanda A. M. Gayer ${ }^{1}$ \\ FT/Unicamp, Limeira, SP \\ Elaine Cristina Catapani Poletti ${ }^{2}$ \\ FT/Unicamp, Limeira, SP \\ André Franceschi de Angelis ${ }^{3}$ \\ FT/Unicamp, Limeira, SP
}

\begin{abstract}
Uma vez que os rios desempenham um importante papel como recursos hídricos, o presente trabalho propõe o estudo da qualidade de água do Rio Jaguari através de modelagem matemática e computacional, utilizando a teoria das redes complexas. Esta pesquisa baseou-se em coletas de amostras de águas do Rio Jaguari, no período de 2006 a 2017, em ponto estratégico na cidade de Paulínia - SP, local densamente povoado e industrializado da região de Campinas, com grande importância econômica para o país. As análises das amostras possibilitaram a criação de um banco de dados atualizado com nove parâmetros importantes: pH, oxigênio dissolvido, demanda bioquímica de oxigênio, nitrogênio total, fósforo total, turbidez, Escherichia coli, sólidos totais e temperatura. Utilizando-se redes complexas, foram analisadas as relações entre os parâmetros amostrados, aplicando a correlação de Pearson. O modelo desenvolvido buscou identificar as variáveis mais impactantes na poluição hídrica do rio durante o período mencionado, tendo-se por base o índice de qualidade da água da Companhia Ambiental do Estado de São Paulo (CETESB), com vistas a fornecer informações de relações entre parâmetros e de subsidiar tomadas de decisões e estudo de estratégias de recuperação da qualidade das águas do rio. Com base na análise dos resultados obtidos, o parâmetro com maior importância é o fósforo total para as qualidades de água aceitáveis, e o nitrogênio total como parâmetro mais significativo para as águas de qualidade ruim. De acordo com este modelo, a densidade da rede aumenta com a piora da qualidade da água, identificando o comportamento da rede de acordo com a qualidade da água analisada.
\end{abstract}

Palavras-chave. Redes Complexas, Modelagem Matemática, Rio Jaguari, Qualidade da Água

\section{Introdução}

A inquietação da sociedade mundial quanto aos problemas ambientais fundamenta-se no esgotamento dos recursos naturais e na falta de controle dos cuidados com o meio ambiente. Dentre estes temas, destaca-se a escassez dos recursos hídricos que são vitais aos seres vivos, principalmente os rios [7].

A menor porção de quantidade de água doce encontrada no planeta é proveniente dos rios. Porém, estes possuem grande importância, uma vez que participam ativamente do desenvolvimento humano. Os rios são fonte de água para consumo, entretenimento, transporte, fonte de alimentos,

\footnotetext{
1f208015@dac.unicamp.br

2 elainec@ft.unicamp.br

3 andre@ft.unicamp.br
} 
fonte de água para indústria e agricultura, além de manter a fauna e a flora do meio ambiente em que estão inseridos $[2,3]$.

Com o crescimento populacional, existe uma degradação qualitativa das águas fluviais, devido à expansão industrial, descarte imprudente de resíduos, mudanças climáticas, alterações no meio ambiente e uso da terra, além de modificações nos fluxos dos rios com criações de barragens. Dessa forma, o estudo das águas dos rios é um tema relevante para o desenvolvimento socioeconômico responsável, garantindo a preservação da natureza $[3,7,11]$.

Este estudo apresenta uma análise de dados do Rio Jaguari que faz parte das bacias dos rios Piracicaba, Capivari e Jundiaí (PCJ) e é de grande importância para o interior do Estado de São Paulo. Além disso, está localizado em uma região de alta industrialização, com um valor econômico e social muito grande para todo o país [6] e apresenta a qualidade da água comprometida, devido ao lançamento de esgoto doméstico e industrial [10].

Entre os anos de 2006 e 2017, foram realizadas coletas de água no rio para análise laboratorial de elementos físicos, químicos e biológicos. Tais coletas foram realizadas e analisadas pela equipe liderada pela Profa. Dra. Dejanira de Franceschi de Angelis ${ }^{4}$, da Universidade Estadual Paulista Julio de Mesquita Filho - UNESP, campus de Rio Claro-SP.

O objetivo geral deste trabalho é analisar, via modelagem de redes complexas, a relação dos parâmetros avaliados nas amostras de águas dos rio, utilizando o índice de qualidade de água (IQA) homologado pela Companhia Ambiental do Estado de São Paulo (CETESB), com vistas a entender e dar indicativos das relações entre os parâmetros avaliados, identificando as variáveis mais impactantes na poluição hídrica do rio durante o período amostrado.

\section{Materiais}

Localizado na bacia do Rio Piracicaba, o Rio Jaguari nasce no Estado de Minas Gerais e participa do sistema de reservatório conhecido como Cantareira que abastece toda a região metropolitana de São Paulo. Além disso, o Rio Jaguari abastece as cidades da região de Campinas. Dessa forma, grande área econômica e industrial do país é dependente deste recurso hídrico [4].

Para estudo do Rio Jaguari, coletas e análises das águas dos rios foram realizadas entre maio de 2006 e janeiro de 2017, aproximadamente quinzenalmente, totalizando 183 amostras. As coletas

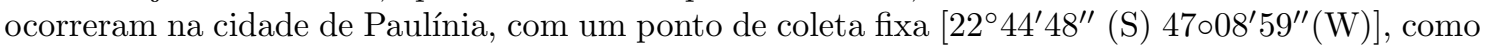
pode ser observada na Figura 1. Todas as coletas foram realizadas pelo amostrador Valdenilson José Alves de Oliveira ${ }^{5}$.

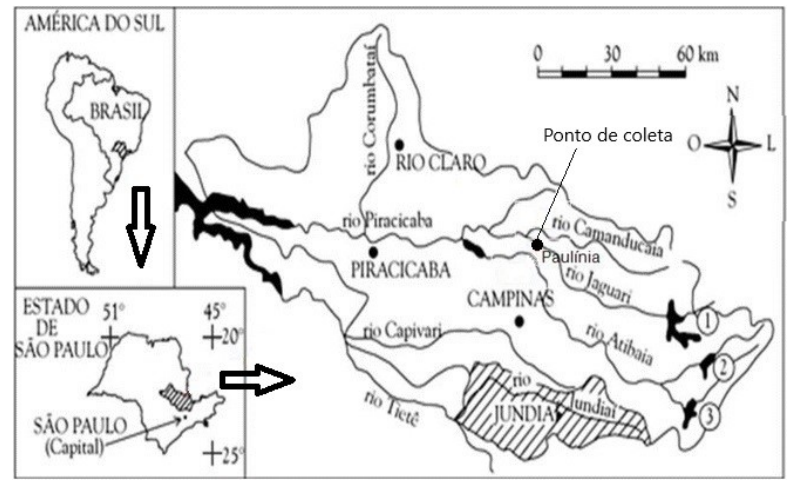

Figura 1: Localização do ponto de coleta. Adaptado de RAMOS et al., 2016, p. 51.

\footnotetext{
${ }^{4}$ http://lattes.cnpq.br/4770174133427369

${ }^{5}$ http://lattes.cnpq.br/3216780233273241
} 
Das amostras, foram analisados 9 parâmetros: quantidade de oxigênio dissolvido (OD), quantidade de Escherichia coli (E. coli), potencial hidrogeniônico $(\mathrm{pH})$, demanda bioquímica de oxigênio (DBO), nitrogênio total ( $\mathrm{N}$ Total), fósforo total (P Total), temperatura, turbidez e sólidos totais dissolvidos (STD).

\section{Métodos}

Para identificar o parâmetro que mais interfere na qualidade da água do rio, a Teoria de Redes Complexas é utilizada como modelo para relacionar os parâmetros do conjunto de dados do rio. A construção das redes leva em consideração a classificação da qualidade da água de cada amostra, utilizando o índice de qualidade da água da Companhia Ambiental do Estado de São Paulo, CETESB.

\section{1 Índice de qualidade da água}

Para identificar as condições da água e interpretar seus parâmetros, pesquisadores propuseram a utilização de cálculos capazes de sintetizar em um único número, resultando em uma única classificação de qualidade, conhecido como índice de qualidade de água, IQA.

A Companhia Ambiental do Estado de São Paulo, CETESB, utiliza o índice IQA desde 1975 como forma de comunicação da qualidade da água para a sociedade e para realizar o gerenciamento dos sistemas hídricos [1].

Os parâmetros analisados pelo IQA da CETESB são: E. coli, pH, DBO, N Total, P Total, turbidez, STD, OD e temperatura. O índice é calculado pelo produtório descrito na Equação (1) $[1]$

$$
I Q A=\prod_{i=1}^{n} w_{i} q_{i}
$$

onde $I Q A$ é o índice de qualidade de água, um número entre 0 e $100, q_{i}$ é a qualidade do i-ésimo parâmetro, também um número entre 0 e 100, obtido das curvas de variação de qualidade, $w_{i}$ é o peso do i-ésimo parâmetro e $n$ é o número de variáveis que participam do cálculo do IQA. O valor do índice IQA CETESB classifica a qualidade da água em análise de acordo com a Tabela 1.

Tabela 1: Classificação da qualidade da água utilizando o índice IQA CETESB [1].

\begin{tabular}{cc}
\hline Classificação & Valores IQA CETESB \\
\hline Ótima & $79<I Q A \leq 100$ \\
Boa & $51<I Q A \leq 79$ \\
Regular & $36<I Q A \leq 51$ \\
Ruim & $19<I Q A \leq 36$ \\
Péssima & $I Q A \leq 19$ \\
\hline
\end{tabular}

\subsection{Redes complexas para modelagem da qualidade da água do Rio Ja- guari}

As redes complexas são compostas por um grupo de itens, chamados de nós, que se conectam através das ligações [5]. Elas podem modelar sistemas reais e a topologia da rede possui grande implicação na análise do comportamento dos sistemas estudados. 
O sistema a ser modelado neste estudo são os parâmetros coletados das amostras do Rio Jaguari. Portanto, os nós da rede são os 9 parâmetros analisados e a ligação entre eles é dado pelo coeficiente de correlação de Pearson. O coeficiente de correlação de Pearson, $r$, determina um grau de relação linear entre duas variáveis [13].

O coeficiente de correlação de Pearson, $r$, são valores entre -1 e 1, para os pesos das ligações são considerados valores em módulo. Para evitar ligações fracas, o valor de 0,3 foi fixado como menor coeficiente capaz de representar a existência de correlação entre duas variáveis.

Dessa forma, o modelo utilizado é o de rede complexa ponderada, ou seja, não se restringe ao fato de existir ou não ligação entre os nós, mas à existência de um valor que define o peso da ligação entre cada nó, como pode ser obervado na Equação (2).

$$
W_{i j}^{w}=\left\{\begin{array}{cl}
\left|r_{(i j)}\right|, & \text { se }\left|r_{(i j)}\right| \geq 0,3 \\
0, & \text { se }\left|r_{(i j)}\right|<0,3
\end{array}\right.
$$

onde $W_{i j}^{w}$ é o valor ponderado da ligação, peso da ligação, entre os nós $i$ e $j$ da rede e $r_{(i j)}$ é o coeficiente da correlação de Pearson entre as variáveis representadas pelos nós $i$ e $j$.

O grau do nó, quantidade de ligações dos nós aos demais, e a força do nó, somatória dos pesos das ligações do nó, são dados importantes para a compreensão das redes complexas. Conhecendo o grau e a força de cada nó, pode-se extrair algumas propriedades estatísticas importantes da rede em análise, como a força média da rede e a sua densidade. A força média de uma rede, dado por $\langle k\rangle$, é a média aritmética das forças de todos os nós da rede. A densidade da rede é a quantidade de ligações que a rede possui em relação a quantidade de possíveis ligações que a rede pode realizar entre os seus nós. A informação da força média e da densidade possibilita compreender a complexidade das ligações dos nós da rede [5].

Para modelar os dados das coletas do Rio Jaguari, os 9 parâmetros das coletas são os nós e as ligações são dadas pela correlação de Pearson. As redes são criadas com os dados das amostras agrupados de acordo com sua classificação da qualidade da água do índice IQA da CETESB.

\section{Resultados e discussões}

Realizando os cálculos de IQA CETESB para cada coleta, foi verificado que, no período considerado, foram encontradas amostras com três classificações: boa, regular e ruim. Todas as amostras foram agrupadas em conjuntos conforme sua classificação. Portanto, três conjuntos foram criados, apresentados na Tabela 2 .

Tabela 2: Tabela de conjuntos de amostras utilizando a classificação do IQA CETESB.

\begin{tabular}{ccc}
\hline Rio & Classificação IQA CETESB & Quantidade de coletas \\
\hline Jaguari & boa & 124 coletas \\
Jaguari & regular & 47 coletas \\
Jaguari & ruim & 12 coletas \\
\hline
\end{tabular}

Foram criadas três redes complexas: uma rede que modela o conjunto de dados das coletas classificadas com qualidade boa (rede $A$ ), uma rede que modela o conjunto de dados das coletas classificadas com qualidade regular(rede $B$ ) e outra que modela o conjunto de dados das coletas classificadas com qualidade ruim (rede $C$ ). As redes foram construídas utilizando os 9 parâmetros das coletas como nós e o coeficiente de correlação de Pearson entre os valores dos parâmetros como valor dos pesos das ligações entre os nós, conforme a Equação (2). A representação visual das redes $A, B$ e $C$ pode ser observada na Figura 2. 


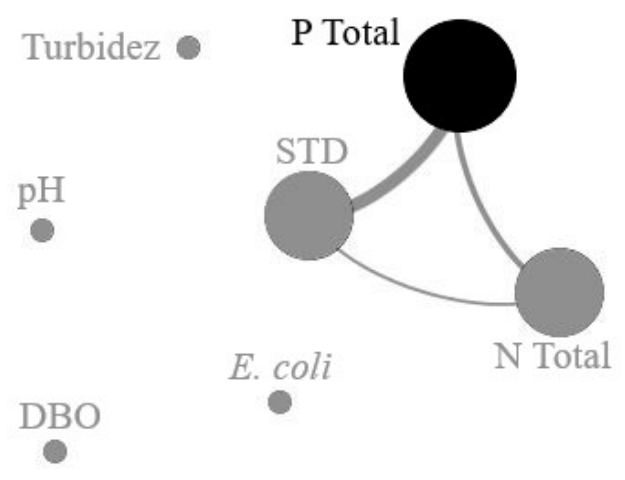

Temperatura P

(a) $A$ - Rede das coletas com IQA bom.

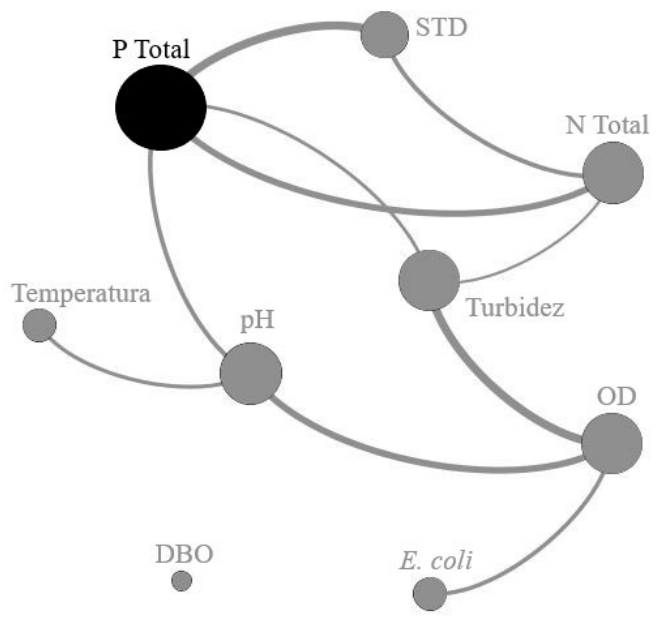

(b) $B$ - Rede das coletas com IQA regular.

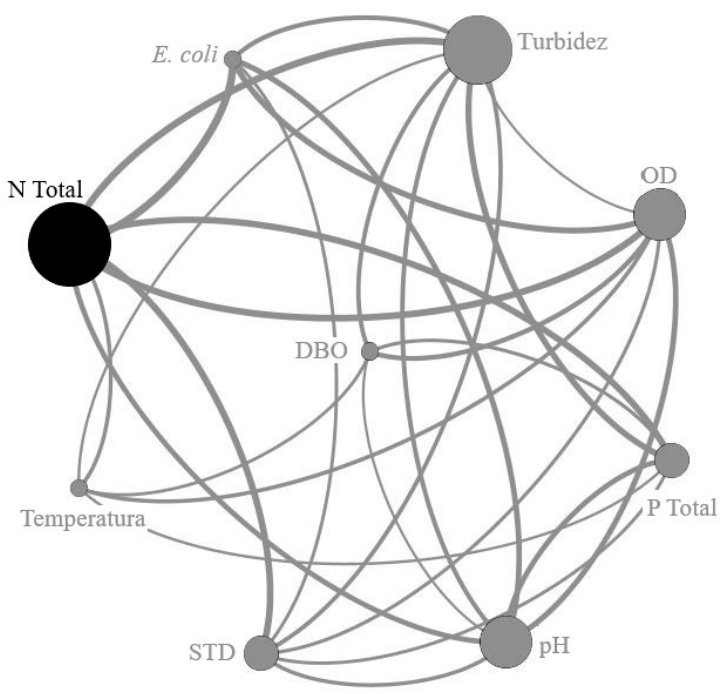

(c) $C$ - Rede das coletas com IQA ruim.

Figura 2: Representações das redes do Rio Jaguari.

A rede $A$, que modela os parâmetros das coletas com classificação de IQA boa, possui uma quantidade de ligações muito baixa, ou seja, possui uma baixa densidade. A força média da rede A é de 0,24 e a densidade é de 0,08. O nó com maior força é o nó que representa o parâmetro de $\mathrm{P}$ Total.

Já a rede $B$, que modela os parâmetros das coletas com classificação de IQA regular, possui uma quantidade de ligações um pouco maior que da rede $A$, porém ainda baixa. O nó com maior força é o nó que representa o parâmetro de $\mathrm{P}$ Total, assim como na rede $A$. A força média da rede $B$ é de 0,84 e a densidade da rede é de 0,28 , ainda baixa, porém maior que a rede $A$. A qualidade de água regular é considerada como aceitável para consumo de acordo com as diretrizes da CETESB.

Diferente das demais redes, a rede $C$, que modela os parâmetros das coletas com classificação 
de IQA ruim, ou seja, água não aceitável para consumo, possui uma grande quantidade de ligações entre seus nós. O nó com maior força é o que representa o parâmetro de N Total.

A rede $C$ possui uma característica diferente das anteriores, não possuindo nenhum nó isolado, ou seja, todos os nós possuem pelo menos uma ligação. A força média da rede $C$ é de 2,88 e a densidade da rede é de 0,78 , muito superiores às métricas das redes $A$ e $B$, conforme pode ser observado na Tabela 3 .

Tabela 3: Tabela de métricas das redes $A, B$ e $C$.

\begin{tabular}{cccc}
\hline & Rede $A$ & Rede $B$ & Rede $C$ \\
\hline Força média & 0,24 & 0,84 & 2,876 \\
Densidade & 0,08 & 0,28 & 0,78 \\
Nó com maior força & P Total & P Total & N Total \\
\hline
\end{tabular}

De acordo com o modelo criado, dentre os parâmetros analisados, $\mathrm{P}$ Total e N Total são os parâmentros que mais influenciam a qualidade da água. Tanto o $\mathrm{P}$ Total quanto o N Total são encontrados normalmente na água dos rios, uma vez que pariticipam dos sistemas biológicos aquáticos. Porém, a poluição pode acarretar uma adição artificial desses componentes químicos, elevando suas concentrações e, consequentemente, aumentando a taxa de crescimento das plantas, conhecida como eutrofização. Do ponto de vista ambiental, as alterações nas concentrações do $\mathrm{P}$ Total e N Total ocasionam alterações em outros parâmetros, como OD e DBO, por causar um desequilíbio ecológico do rio [8].

\section{Conclusões}

A importância dos recursos hídricos como fonte de água doce para sobrevivência dos seres vivos é inquestionável e conhecer a dinâmica e os sistemas que envolvem a qualidade da água dos rios torna-se, desta forma, fundamental. Dada a importância do Rio Jaguari no interior do Estado de São Paulo, este trabalho estudou dados coletados do mesmo.

O objetivo principal deste estudo, a modelagem dos dados do rio utilizando redes complexas, proporcionou a descoberta dos principais parâmetros que influenciam a qualidade da água, $\mathrm{P}$ Total paras as coletas com qualidade aceitável e N Total para qualidade ruim da água do rio. Tanto o P Total quanto o N Total, participam ativamente nos processos biológicos, porém, quando um ou outro ou ambos encontram-se em abundância, acarretam um crescimento excessivo de plantas aquáticas. O excesso desses parâmetros é proveniente de efluentes domésticos, agrícolas e industriais [9] e é uma preocupação para os os órgãos responsáveis na gestão das águas dos rios, principalmente em áreas com intensas atividades urbanas e agrícolas [12].

A qualidade da água aceitável, boa e regular, apresentou uma baixa linearização entre os 9 parâmetros analisados, apresentando algumas ligações de relação entre alguns parâmetros com o $\mathrm{P}$ Total. Já a qualidade da água ruim apresentou uma alta linearização entre os 9 parâmetros, ou seja, existe uma relação maior dos parâmetros entre si, principalmente com o N Total. Diante disso, a modelagem possibilitou a verificação de um aumento da densidade da rede com a piora da qualidade da água.

\section{Agradecimentos}

O presente trabalho foi realizado com apoio da Coordenação de Aperfeiçoamento de Pessoal de Nível Superior - Brasil (CAPES) - Código de Financiamento 001. 


\section{Referências}

[1] Companhia Ambiental do Estado de São Paulo (CETESB). Relatório de Qualidade das Águas Interiores do Estado de São Paulo - Apêndice D: Índice de Qualidade das Águas, 2017, São Paulo.

[2] Firth, P. The importance of water resources education for the next century, Journal of the American Water Resources Association, 35:487-492, 1999. DOI: 10.1111/j.17521688.1999.tb03605.x.

[3] Grill, G. et al. Mapping the world's free-flowing rivers, Nature, 569:215-221, 2019. DOI: 10.1038/s41586-019-1111-9.

[4] Hoeffel, J. L., Fadini, A. A., Machado, M. K. e Reis, J. C. Trajetórias do Jaguary unidades de conservação, percepção ambiental e turismo: um estudo na APA do Sistema Cantareira, São Paulo, Ambiente \& sociedade, 11:131-148, 2008. DOI: 10.1590/S1414-753X2008000100010.

[5] Mainstone, C.P., Parr, W. Phosphorus in rivers - ecology and management. Science of the Total Environment, 282:25-47, 2002. DOI:10.1016/S0048-9697(01)00937-8.

[6] Newman, M. E. J. The structure and function of complex networks, SIAM review, 45:167-256, 2003. DOI: $10.1137 /$ S003614450342480.

[7] Ramos, M. A. G., Oliveira, E. S. B. de, Pião, A. C. S., Leite, D. A. N. de O. and Angelis, D. de F. de. Water quality index (WQI) of Jaguari and Atibaia Rivers in the region of Paulínia, São Paulo, Brazil, Environmental monitoring and assessment, 188:263-267, 2016. DOI:10.1007/s10661-016-5261-z.

[8] Rosa, A. H., Fraceto, L. F., Moschini-Carlos, V. and Organizadores. Meio ambiente e sustentabilidade, 1a. edição. Bookman, Porto Alegre, 2012. ISBN: 8540701960.

[9] Sawyer, C.N. and Mccarty, P.L. Chemistry for Environmental Engineers, 3rd ed. Mc Graw-Hill Book Company, New York, 1978.

[10] Sistema Integrado de Gerenciamento de Recursos Hídricos do Estado de São Paulo (Sigrh). Situação dos recursos hídricos no estado de São Paulo 2017. Secretaria de Infraestrutura $e$ Meio Ambiente, Governo do Estado de São Paulo. São Paulo, 2019.

[11] Sundaray, S. K., Panda, U. C., Nayak, B. B. and Bhatta, D. Multivariate statistical techniques for the evaluation of spatial and temporal variations in water quality of the Mahanadi riverestuarine system (India)-a case study, Environmental Geochemistry and Health, 28:317-330, 2006. DOI: 10.1007/s10653-005-9001-5.

[12] Weigelhofer, G., Hein, T., Bondar-Kunze, E. Phosphorus and Nitrogen Dynamics in Riverine Systems: Human Impacts and Management Options. Riverine Ecosystem Management: Science for Governing Towards a Sustainable Future. Springer International Publishing, 187-202, Cham, 2018, DOI:10.1007/978-3-319-73250-3_10.

[13] Zhu, H., You, X. and Liu, S. Multiple Ant Colony Optimization Based on Pearson Correlation Coefficient, IEEE Access, 7:61628-61638, 2019. DOI: 10.1109/access.2019.2915673. 